\title{
Alpha-lipoic acid and vitamin B complex slow down the changes in mice diabetic cardiomyopathy
}

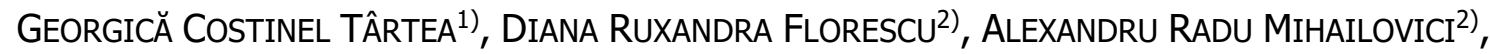 \\ IONUT DONOIU ${ }^{2)}$, OCTAVIAN ISTRĂTOAIE ${ }^{2)}$
}

1) Department of Physiology, University of Medicine and Pharmacy of Craiova, Romania

2) Department of Cardiology, University of Medicine and Pharmacy of Craiova, Romania

\begin{abstract}
Aim: The aim of our study was to assess histologically and by cardiac ultrasound the effects of alpha-lipoic acid (ALA) and vitamin B complex, as pathogenic therapies, in diabetic cardiomyopathy (DCM) in mice. Materials and Methods: We performed an experimental animal study, in which we analyzed from a structural and functional point of view the changes produced in DCM. To produce DCM, we induced diabetes mellitus (DM) in C57BL/6 mice by intraperitoneal injection of a single $150 \mathrm{mg} / \mathrm{kg}$ body weight dose of streptozotocin (STZ). We formed a sham group (animals without DM), a control group (animals with DM but without treatment, DM_Control) and a group of animals with DM that were treated with ALA and vitamin B complex (DM_Treated). Results: At six weeks after STZ administration, there was no decrease in left ventricular ejection fraction (LVEF) in the sham group, while in the control group there was a significant decrease in LVEF, about $43.75 \pm 3.37 \%$, compared to the group that received treatment with ALA and vitamin B complex, in which LVEF decreased to $49.6 \pm 5.02 \%(p=0.0432)$. Also, the degree of interstitial myocardial fibrosis was higher in animals with DM compared to animals without DM, but the applied therapeutic protocol considerably improved the accumulation of interstitial collagen. The same observation was maintained regarding the evaluation of polysaccharide deposits. Conclusions: We can say that the administration of ALA and vitamin B complex in mice with STZ-induced DM, improves the degree of myocardial fibrosis, the accumulation of polysaccharides, and prevents severe deterioration of systolic and diastolic function of the heart.
\end{abstract}

Keywords: diabetes mellitus, streptozotocin, diabetic cardiomyopathy.

\section{Introduction}

Diabetes affects approximately 463 million people worldwide, and this number is projected to reach approximately 700 million people by 2040 due to urbanization, population aging, adverse dietary changes and reduced physical activity $[1,2]$. This disease affects almost all tissues in the body, thus increasing morbidity and mortality due to considerable organ dysfunction, especially the heart. Thus, cardiovascular disease is responsible for approximately $65 \%$ of diabetes-related deaths $[3,4]$. Numerous clinical studies show that diabetes is an important risk factor for acute myocardial infarction [1-4]. Similarly, diabetes increases the risk of heart failure by up to five times [4]. However, the increased prevalence of heart failure in diabetics cannot be explained by concomitant hypertension and coronary heart disease. Diabetic cardiomyopathy (DCM) is recognized as one of the main complications of diabetes which, regardless of the presence of coronary heart disease or hypertension, increases the risk of heart failure and death $[4,5]$.

Numerous studies evaluate the structural and functional molecular mechanisms and possible therapeutic targets for the prevention and treatment of DCM [4]. The main mechanisms involved in the initiation and progression of DCM are hyperglycemia, oxidative stress, increased insulin resistance, left ventricular (LV) hypertrophy, impaired systolic and diastolic functions of the left ventricle, myocardial fibrosis, cardiomyocyte death, autophagy, endothelial dysfunction and endoplasmic reticulum stress [5-7].

This disease was first described by Rubler et al., in 1972, who reported information found at the autopsy performed on diabetic patients with LV dilation in the absence of any proven etiology of heart failure [6]. Subsequent studies confirmed the initial observation, resulting in the concept of DCM. The main feature of DCM is represented by diastolic dysfunction in the absence of systolic dysfunction, but also the high degree of myocardial fibrosis in the absence of hypertension or coronary artery disease [8-13].

Despite the known relationship between altered energy metabolism and increased insulin resistance in patients with diabetes, the mechanism underlying the initiation and progression of DCM remains to be elucidated. Thus, in addition to adequate glycemic control, potential therapies to prevent DCM may be represented by alpha-lipoic acid (ALA) and vitamin B complex (thiamine and pyridoxamine). Alpha-lipoic acid (also called thioctic acid) is known as a cofactor for alpha-ketoacid mitochondrial dehydrogenases and has been shown to play an important role in the energy metabolism of mitochondria [14, 15]. ALA increases glucose utilization in isolated rat hearts [16]. Other studies suggest that ALA causes a cellular antioxidant state by improving the action of antioxidant enzymes [17]. ALA administration

This is an open-access article distributed under the terms of a Creative Commons Attribution-NonCommercial-ShareAlike 4.0 International Public License, which permits unrestricted use, adaptation, distribution and reproduction in any medium, non-commercially, provided the new creations are licensed under identical terms as the original work and the original work is properly cited. 
reduces the content of advanced glycation end products (AGEs), but also the production of mitochondrial superoxide in cardiomyocytes and decreases insulin resistance in diabetic animal models [18]. On the other hand, thiamine and pyridoxine, $\mathrm{B}$ vitamins, have multiple roles in the body, including those to prevent various molecular signaling pathways altered by increased plasma glucose levels [19-21].

\section{Aim}

The aim of our study was to evaluate histologically and through echocardiography the effects of ALA and vitamin B complex, as pathogenic therapies, in DCM in mice.

\section{ㅁ Materials and Methods}

\section{Animal model}

We performed an experimental animal study, in which we analyzed from a structural and functional point of view the changes produced in DCM. It must be said that all the institutional guidelines for experiments on laboratory animals were followed and we obtained the approval of the Ethics Committee of the University of Medicine and Pharmacy (UMPh) of Craiova. The UMPh of Craiova Animal Facility, in which the study was place, operates under Federation of European Laboratory Animal Science Associations (FELASA) accreditation. All procedures involving animals complied to Standards Relating to the Care and Management of Experimental Animals in research and other regulations. This study complies to the relevant national, European Union (EU) and international ethicsrelated rules and professional codes of conduct. All experiments were performed in accordance with the European Council Directive (86/609/EEC). Also, the anatomopathological analysis was performed in the Research Center for Microscopic Morphology and Immunology from UMPh of Craiova.

We housed the experimental animals (C57BL/6 mice) in a pathogen-free environment, with continuous access to food and water, in a program of 12 hours of light, 12 hours of darkness. Plasma glucose levels were determined at the enrollment of animals in the study, before the induction of diabetes mellitus (DM), subsequently every week in order to document the onset and duration of DM. Blood glucose was determined by taking blood from a large vein in the animals' tail by using a standard glucometer (Contour Plus One, Ascensia Diabetes Care, Switzerland).

For the DCM study, we induced DM in C57BL/6 mice by intraperitoneal injection of a single $150 \mathrm{mg} / \mathrm{kg}$ body weight (b.w.) dose of streptozotocin (STZ).

The experiments were performed on 24 C57BL/6 mice, male and female, aged between eight weeks and 10 weeks, weighing between $20 \mathrm{~g}$ and $27 \mathrm{~g}$. Before performing echocardiographic recordings or collecting blood, the animals were removed from the animal care complex, where they had, as already mentioned, food and water ad libitum.

The animals were randomly divided into three distinct groups, as follows:

(i) Sham group, in which only $0.9 \%$ sodium chloride was injected, without STZ being injected, so without
DM. The purpose of this group was to examine cardiac echocardiographic and histological changes and also biological changes (weight, plasma glucose, etc.) in the absence of DM.

(ii) Control group injected with STZ $150 \mathrm{mg} / \mathrm{kg}$ b.w., intraperitoneally, single administration, at 8-10 weeks of age. No therapy was applied to this group. In this case, the biological parameters of the animals were observed (weight, diuresis, polydipsia, polyphagia) but cardiac structural parameters were also evaluated (by echocardiography and histological analysis).

(iii) Treated group with therapeutic dose of alpha thioctic acid and vitamin B complex (abbreviated A+B). At this animal site, after inducing DM by injecting intraperitoneal STZ and confirming the presence of DM by high blood glucose but also the occurrence of polyuria, polyphagia and polydipsia, the therapeutic protocol was applied both by intraperitoneal administration of alphathioctic acid (by intraperitoneal administration of $100 \mathrm{mg} / \mathrm{kg}$ b.w. of ALA - Thiossen, Solupharm Pharmazeutische Erzeugnisse GmbH, Melsungen, Germany) and also of the vitamin B complex [thiamine hydrochloride (vitamin B1) $100 \mathrm{mg} / \mathrm{kg}$ b.w. and pyridoxine hydrochloride (vitamin B6) $50 \mathrm{mg} / \mathrm{kg}$ b.w. - Milgamma ${ }^{\circledR}$, Wörwag Pharma, Böblingen, Germany]. In order to avoid acute water intoxication of the animals, we decided to initially administer the dose of ALA (approximately $200 \mu \mathrm{L}$ per animal) and then, at an interval of at least six hours, the dose of vitamin B complex (approximately $100 \mu \mathrm{L}$ per animal). The therapy was applied five days a week for six weeks.

\section{Cardiac function measurement}

To perform structural and functional cardiac measurements, we used an Ultrasonix ultrasound machine (Sonix Touch version, Richmond, Canada) equipped with a linear probe for small animals but also with a special software for cardiac analysis (L40-8/12, bandwidth 8-40 MHz, depth range $0.1-3.0 \mathrm{~cm})$. For echocardiographic analysis, the animals were anesthetized with a cocktail of Ketamine $(50 \mathrm{mg} / \mathrm{mL}) /$ Xylazine $(7 \mathrm{mg} / \mathrm{mL})$, which allowed a good frame rate as the heart rate decreased during the analysis to about 150 beats/minute. M-mode, two-dimensional (2D) and pulsed-wave Doppler echocardiography were used to assess left ventricular ejection fraction (LVEF), fractional shortening (FS), left ventricular end-diastolic diameter (LVEDd), peak velocity of early (E) and late (A) ventricular filling velocity, ratio of early to late mitral inflow velocity (E/A), and LV internal diameter at endsystole. LVEDd and thickness of the septum and posterior wall were determined from the long-axis view. E and A waves were obtained by the apical four-chamber view at the level of mitral inflow by pulsed-wave Doppler.

\section{Histology}

After euthanizing the animals under deep anesthesia, the heart was dissected and immediately included in potassium chloride, in order to be stopped in diastole. Subsequently, it was fixed in 4\% formalin for 24 hours. After the 24 hours to 48 hours in which the hearts were fixed, they were washed for 24 hours, in order to remove the formalin-fixing solution from the tissues, after which 
they were included in paraffin, in order to perform serial sections of $3-5 \mu \mathrm{m}$ thick, which could be optimally stained and analyzed with an optical microscope. After obtaining the tissue blocks, serial sections of $3 \mu \mathrm{m}$ thickness were made using a high-precision HM355S automatic rotary microtome, equipped with a section transfer system initially on a cold water bath and then transferred to a warm $\left(40^{\circ} \mathrm{C}\right)$ water bath, in order to be stretched and uniformed. Sections obtained were then collected and glued on poly-L-lysine slides and placed in an incubator, at $60^{\circ} \mathrm{C}$, and stored for 24 hours. Tissue sections, used in our study, were stained first by using the Hematoxylin-Eosin (HE) staining technique. To examine cardiac fibrosis, heart samples were stained with Masson's trichrome and in order to assess extracellular matrix (ECM) deposition of polysaccharides, we used Periodic Acid-Schiff (PAS) staining. The level of polysaccharides staining received a score that varied from one cross $(+)$, representing weak reaction for glycogen, to four crosses $(++++)$, representing the strongest staining for glycogen $[22,23]$. Slides were assessed on a Nikon 55i microscope (Apidrag, Bucharest, Romania), equipped with a 5-megapixel color cooled charge-coupled device (CCD) camera and the Image-Pro Plus AMS 7 software (Media Cybernetics, Rockville, MD, USA). With the help of the latter software, the quantitative analysis of myocardial fibrosis was performed by analyzing 10 randomized images for each slice.

\section{Statistical analysis}

All data were expressed as mean and standard deviation. Initially, the data were exported to Microsoft Office Excel 2019 (Microsoft Corporation, Redmond, Washington, USA) and, after the graphical representation, were exported to GraphPad software (version 9.0, CA, USA). We used the two-way analysis of variance (ANOVA) test to assess the statistical differences between the means of more than two data groups. In all cases where we had the value of $p<0.05$, we considered a statistically significant difference between the means of the compared groups. Moreover, the value of $p<0.05, p<0.01$ and $p<0.001$ represented a statistically significant, highly significant, and very highly significant difference.

\section{吕 Results}

DM was induced in 24 animals randomly divided into three groups. As compared with sodium chloride treatment, STZ induced rapid hyperglycemia in mice beginning at 4-5 days after intraperitoneal injection (Figure 1A). Thus, at enrollment in the study, the plasma glucose level was $107.16 \pm 16.01 \mathrm{mg} / \mathrm{dL}$ for the sham group, 113.16 $\pm 27.73 \mathrm{mg} / \mathrm{dL}$ for the group of animals with DM, but without treatment (DM_Control) and $119.23 \pm 16.56 \mathrm{mg} / \mathrm{dL}$ in the group of animals with DM but treated with ALA and vitamin B complex (DM_Treated) without any statistically significant differences between the groups of animals enrolled in the study. At six weeks after the enrollment of the studied animals, the plasma blood glucose level in the sham group was $126.83 \pm 37.92 \mathrm{mg} / \mathrm{dL}$, in the DM_Control group $548.16 \pm 49.23 \mathrm{mg} / \mathrm{dL}$ and in the DM_Treated group of $570.56 \pm 36.12 \mathrm{mg} / \mathrm{dL}$, statistically significant differences were registered both between the sham group and DM_Control $(p=0.0001)$ but, also, between the sham group and the DM_Treated group $(p<0.0001)$, while between the DM_Control and DM_Treated groups, no statistically differences were registered as significant $(p=0.5702)$. Another important aspect in this study was the variation of the body weight of the enrolled animals. Thus, at the time of enrollment, we observed a weight of $23.42 \pm 1.19 \mathrm{~g}$ in the sham group, in the DM_Control group of $25.71 \pm 2.14 \mathrm{~g}$, and in the DM_Treated group of $24.16 \pm 2.78 \mathrm{~g}$.

Thus, we analyzed the percentage variation of body weight after the administration of STZ and we observed that in the case of animals without DM (sham), there was an increase (at six weeks of approximately $8.4 \pm 3.9 \%$ ), while in the DM groups there was a decrease (at six weeks of approximately $20.21 \pm 4.66 \%$ for the DM_Control group and $24.64 \pm 3.36 \%$ for the DM_Treated group), noticing statistically significant differences between sham group and DM_Control $(p=0.0007)$ and DM_Treated $(p=0.004)$, while between DM_Control and DM_Treated, there were no statistically significant differences $(p=0.1294)$.

Animals enrolled in our study were evaluated by cardiac ultrasound before STZ administration but also six weeks after enrollment, before being euthanized. The determined echocardiographic parameters were highlighted in Table 1. First of all, we analyzed the LVEF, as shown in Figure 2. It should be mentioned that all evaluated parameters were obtained by 2D echocardiography [LVEF, LVEDd, left ventricular end-systolic diameter (LVESd), interventricular septum in diastole (IVS), left ventricular posterior wall thickness (LVPW)], M mode (FS) and pulsed Doppler (E, $\mathrm{A}$ and $\mathrm{E} / \mathrm{A}$ ratio), as shown in Figure 3. At enrollment of the studied animals, there were no statistically significant differences between different groups. At six weeks after STZ administration in the sham group, there were no decreases in ejection fraction, while at six weeks after STZ administration in the control group, there was a significant decrease in LVEF to approximately $43.75 \pm 3.37 \%$ compared to the group that received treatment with ALA and vitamin B complex, in which LVEF decreased to $49.6 \pm 5.02 \%(p=0.0432)$. Also, the decrease in FS was higher in DM_Control compared to DM_Treated $(p=0.0438)$. At six weeks after the induction of DM by STZ, there was an increase in the thickness of the interventricular septum and of the posterior wall of the left ventricle compared to the sham group, but this increase was not slowed down in animals that were treated differently compared to those in the control group $(p=0.715)$. Regarding the diastolic profile evaluated by $\mathrm{E}$ and $\mathrm{A}$ waves at the mitral inflow level, we observed an alteration of myocardial relaxation by decreasing the E wave velocity and increasing the A wave velocity, thus decreasing the E/A ratio, attenuated by the administration of the therapeutic protocol.

We analyzed histologically the degree of myocardial fibrosis by staining collagen fibers (Figure 4, A-C). In the absence of treatment, six weeks after the onset of DM, the degree of myocardial fibrosis in the control group was approximately $5.679 \pm 0.852 \%$, while in the control group, it was $4.318 \pm 0.788 \%$ ( $p=0.0145)$, compared to sham group 
where a degree of myocardial fibrosis of approximately $1.28 \pm 0.473 \%$ was recorded $(p<0.000$; Figure $4 \mathrm{E})$. We also analyzed the extracellular accumulation of polysaccharides by PAS staining (Figure 4D) and found that in the case of diabetic animals (DM_Control) without treatment, there was a higher extracellular accumulation of polysaccharides $(++++)$ compared to diabetic animals but treated with

Plasma glucose levels in the animals enrolled in the study

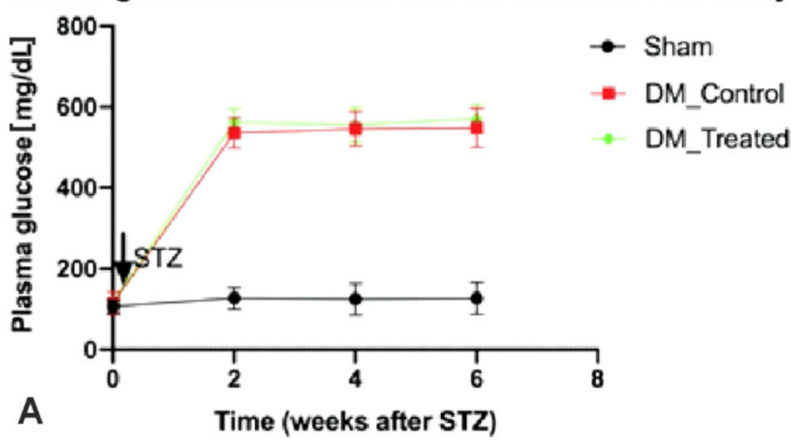

ALA and vitamin B complex $(++,+++)$, thus registering the statistically significant difference $(p=0.0385)$. Also, comparing the sham group with the DM_Control and DM_Treated groups, we found a significant increase in extracellular polysaccharide deposits in the last two groups, especially perivascular $(p<0.000$; Figure $4 \mathrm{~F})$.

The weight of the animals enrolled in the study

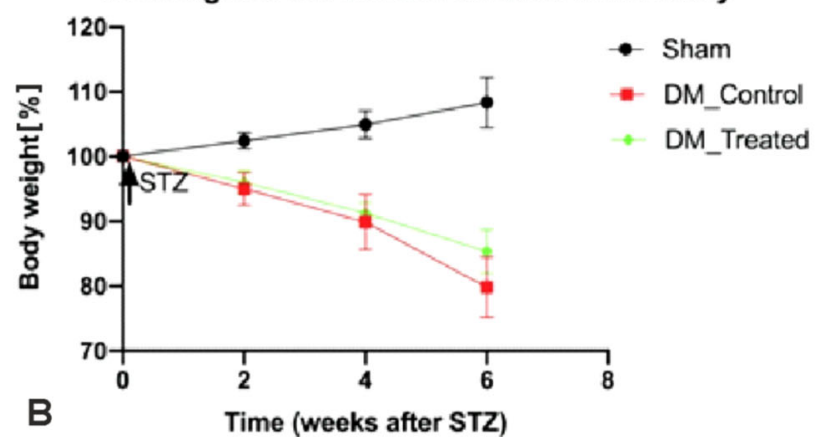

Figure 1 - (A) Plasma glucose levels in the animals enrolled in the study after STZ administration. (B) Percentage change in body weight of animals enrolled in the study after STZ administration. DM: Diabetes mellitus; STZ: Streptozotocin.

Table 1 - Echocardiography parameters in the groups of animals enrolled in our study

\begin{tabular}{ccccccc}
\hline \multirow{2}{*}{ Groups } & \multicolumn{3}{c}{ 0 week (at enrollment) } & \multicolumn{2}{c}{ Six weeks after enrollment } \\
\cline { 2 - 7 } & Sham & $\boldsymbol{D} \boldsymbol{M}_{-}$Control & DM_Treated & Sham & DM_Control & DM_Treated \\
\hline LVEF [\%] & $74.25 \pm 5.4$ & $73.62 \pm 3.99$ & $73.55 \pm 3.71$ & $74.58 \pm 3.62^{\mathrm{a}, \mathrm{b}}$ & $43 \pm 75^{\mathrm{a}, \mathrm{c}}$ & $49.62 \pm 5.01^{\mathrm{b}, \mathrm{c}}$ \\
\hline FS [\%] & $47.79 \pm 5.98$ & $46.75 \pm 5.92$ & $48.25 \pm 5.42$ & $48.38 \pm 4.18^{\mathrm{a}, \mathrm{b}}$ & $36.86 \pm 6.12^{\mathrm{a}, \mathrm{c}}$ & $43.1 \pm 3.44^{\mathrm{b}, \mathrm{c}}$ \\
\hline LVEDd [mm] & $3.64 \pm 0.46$ & $3.59 \pm 0.4$ & $3.75 \pm 0.44$ & $3.68 \pm 0.46^{\mathrm{a}}$ & $3.2 \pm 0.4^{\mathrm{c}}$ & $3.5 \pm 0.44^{\mathrm{c}}$ \\
\hline LVESd [mm] & $1.96 \pm 0.32$ & $1.92 \pm 0.34$ & $1.95 \pm 0.35$ & $1.95 \pm 0.36$ & $2.01 \pm 0.34$ & $1.93 \pm 0.31$ \\
\hline IVS [mm] & $0.86 \pm 0.07$ & $0.84 \pm 0.08$ & $0.87 \pm 0.07$ & $0.89 \pm 0.06^{\mathrm{a}}$ & $1.1 \pm 0.08^{\mathrm{a}}$ & $1.08 \pm 0.07^{\mathrm{a}}$ \\
\hline LVPW [mm] & $0.87 \pm 0.09$ & $0.84 \pm 0.12$ & $0.88 \pm 0.08$ & $0.89 \pm 0.09^{\mathrm{a}}$ & $1.15 \pm 0.12^{\mathrm{a}}$ & $1.11 \pm 0.14^{\mathrm{a}}$ \\
\hline E [cm/s] & $67.25 \pm 5.49$ & $65.5 \pm 3.58$ & $67.25 \pm 5.95$ & $68.5 \pm 5.37^{\mathrm{a}, \mathrm{b}, \mathrm{c}}$ & $30.5 \pm 4.59^{\mathrm{a}, \mathrm{b}, \mathrm{c}}$ & $43.62 \pm 6.45^{\mathrm{a}, \mathrm{b}, \mathrm{c}}$ \\
\hline A [cm/s] & $28.5 \pm 4.12$ & $27.5 \pm 3.62$ & $27.6 \pm 3.85$ & $29.37 \pm 3.66^{\mathrm{a}}$ & $31.5 \pm 3.62^{\mathrm{a}}$ & $30.5 \pm 3.85^{\mathrm{a}}$ \\
\hline E/A & $2.39 \pm 0.33$ & $2.41 \pm 0.47$ & $2.33 \pm 0.35$ & $2.15 \pm 0.32^{\mathrm{a}, \mathrm{b}, \mathrm{c}}$ & $0.97 \pm 0.15^{\mathrm{a}, \mathrm{b}, \mathrm{c}}$ & $1.44 \pm 0.24^{\mathrm{a}, \mathrm{b}, \mathrm{c}}$ \\
\hline
\end{tabular}

LVEF: Left ventricular ejection fraction; FS: Fractional shortening; LVEDd: Left ventricular end-diastolic diameter; LVESd: Left ventricular endsystolic diameter; IVS: Interventricular septum in diastole; LVPW: Left ventricular posterior wall thickness; E: Early wave in diastole; A: Latter wave caused by atrial systole; E/A: E wave to A wave ratio. Two-way ANOVA test: ${ }^{a} p<0.05$ Sham vs. DM_Control; ${ }^{b} p<0.05$ Sham vs. DM_Treated; ${ }^{c} p<0.05$ DM_Control vs. DM_Treated. ANOVA: Analysis of variance; DM: Diabetes mellitus.
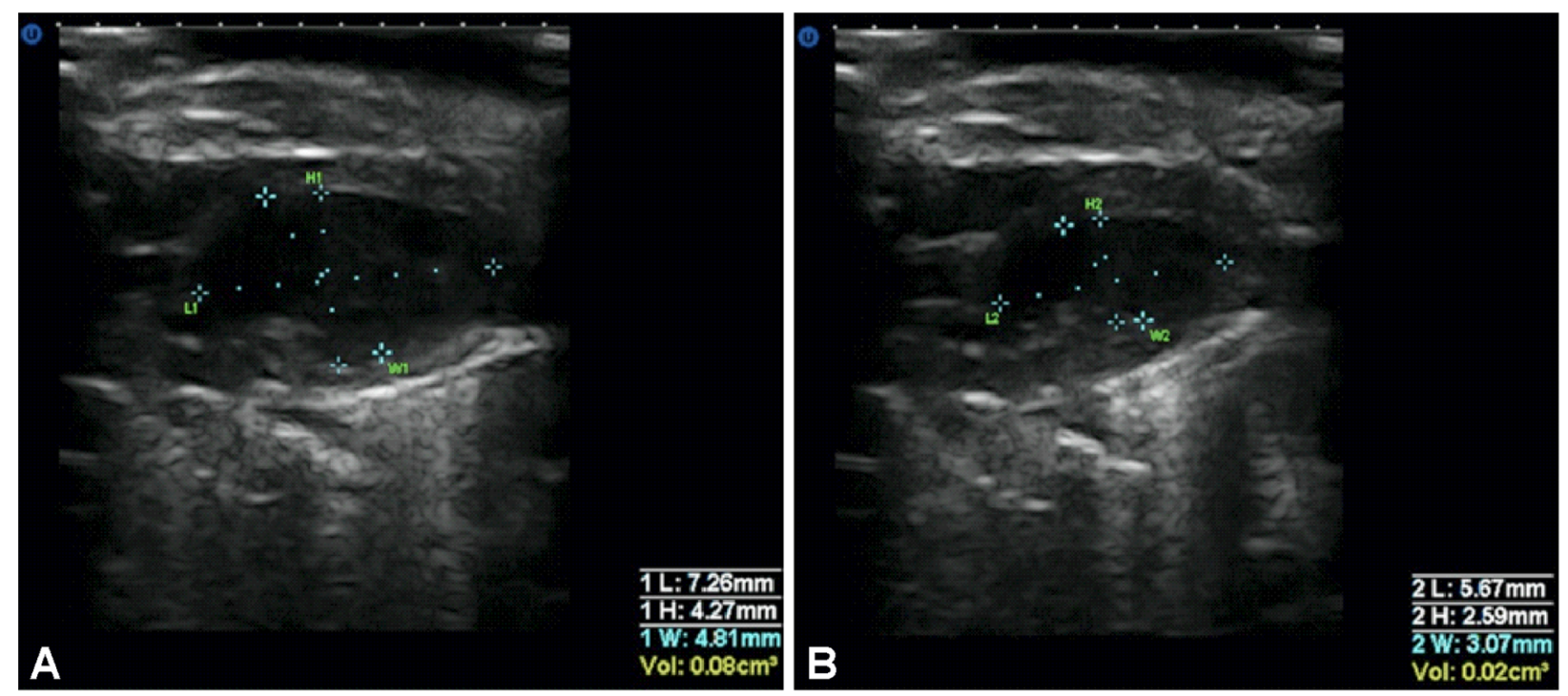

Figure 2 - Example of determining a LVEF: (A) Parasternal long axis view with determination of an end-diastolic volume of $0.08 \mathrm{~cm}^{3}$; (B) Parasternal long axis view with the determination of an end-systolic volume of $0.02 \mathrm{~cm}^{3}$, thus resulting in an LVEF of 75\%. LVEF: Left ventricular ejection fraction. 


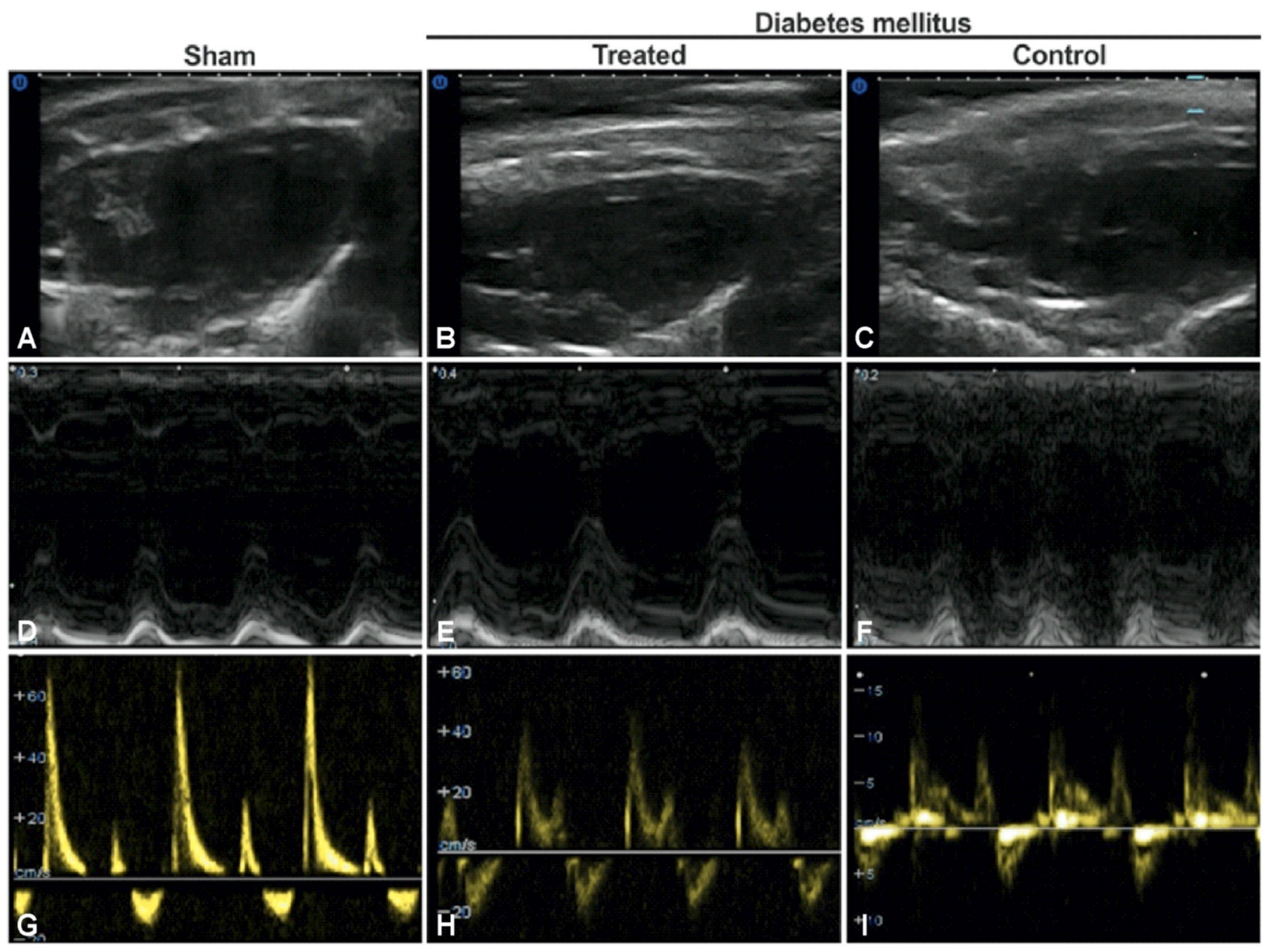

Figure 3 - Echocardiographic assessment of mouse heart: $(A-C)$ Representative 2D echocardiograms; (D-F) Representative M-mode echocardiograms; (G-I) Representative pulsed-wave Doppler echocardiograms of mitral inflow. 2D: Twodimensional.

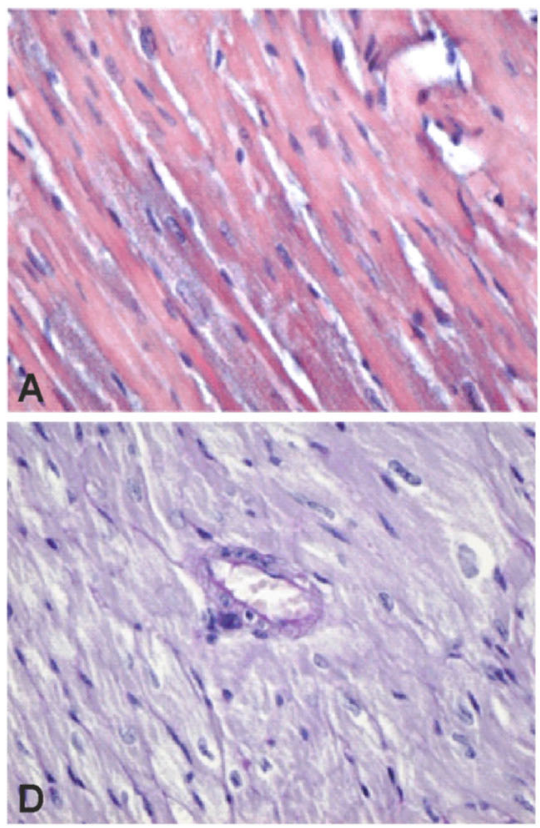

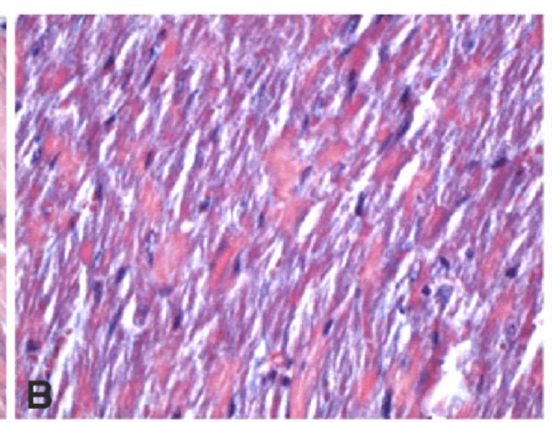

The degree of interstitial myocardial fibrosis for the studied animals

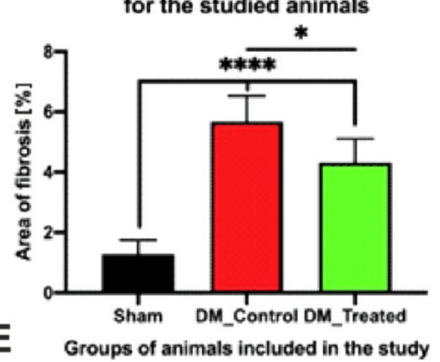

E

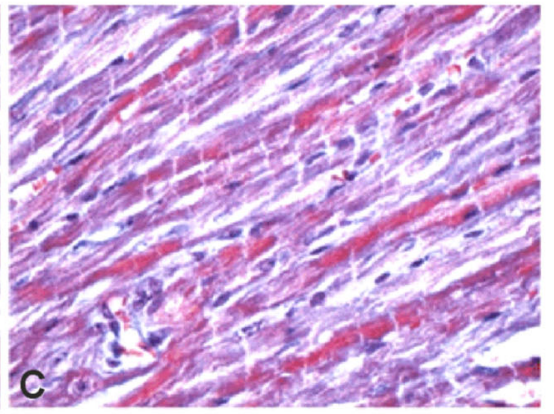

The degree of interstitial deposits with polysaccharides

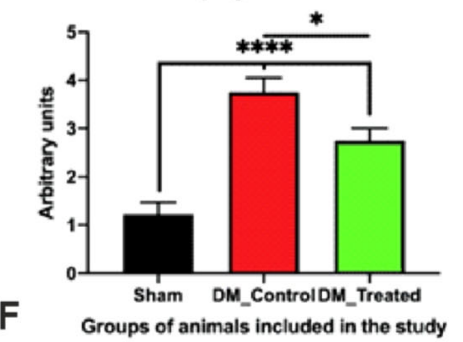

Figure 4 - Representative Masson's trichrome staining $(\times 400)$ in sham (A), DM_Treated (B), and DM_Control (C) groups; (D) PAS staining (×400) for polysaccharide in STZ-induced diabetic mice; (E and F) Quantitative analysis of myocardial fibrosis, respectively polysaccharide deposition: ${ }^{*}<<0.05$, ***p<0.000. DM: Diabetes mellitus; PAS: Periodic Acid-Schiff; STZ: Streptozotocin. 


\section{ㅁ Discussions}

In patients with diabetes, one cause of death is heart failure, which is caused in part by DCM. Because the role of oxidative stress is extremely important in the pathogenesis of DCM, antioxidant therapies, such as ALA, have been analyzed in this disease. Our study explores the protective role of the administration of ALA and vitamin B complex in the STZ-induced diabetic mice model.

The most commonly used model of DM is the STZ model. It is a structurally similar antibiotic to glucose and is preferentially taken up by the glucose transporter 2 (GLUT2) in insulin-secreting pancreatic beta-cells [13, $24,25]$. Intraperitoneal administration of STZ causes betacell necrosis and subsequently insulin deficiency. Many studies performed on mice with STZ-induced DM have shown the occurrence of systolic and diastolic insufficiency, their degree being proportional to the duration of DM [13, 25]. DM can also be induced at different ages, allowing the study of its effects on the heart at different stages of animal life [25].

Interstitial collagen accumulation and subsequent myocardial fibrosis are essential structural changes that occur in DCM. Diabetes locally causes activation of the renin-angiotensin-aldosterone system (RAAS), which causes cardiomyocyte necrosis and interstitial fibrosis $[26,27]$. Fibrous tissue in myocardium can be located interstitially or perivascular, or together, and histopathological analysis identifies capillary endothelial changes, interstitial fibrosis, thickening of the capillary basal lamina, and finally myocardial hypertrophy. Structural changes in DCM are related to cardiac remodeling, consisting of LV hypertrophy, but also systolic and diastolic dysfunction. LV hypertrophy is usually demonstrated by a more advanced phase of DCM [27].

Alpha-lipoic acid (or thioctic acid) is an antioxidant and a natural constituent involved in mitochondrial dehydrogenation reactions. Experimental studies have confirmed that intraperitoneal administration of thioctic acid to Wistar rats with STZ-induced DM has normalized thiobarbituric acid levels in the retina, pancreas, liver, and plasma [13]. In addition, in cardiovascular disease, the administration of thioctic acid has been used effectively in various in vivo models, such as heart failure, hypertension or ischemia/reperfusion models [28]. Chun et al. reported that administration of thioctic acid to DCM rats showed myocardial dysfunction, increased heart rate, interstitial collagen deposition; they also found that the degree of cardiac fibrosis was positively correlated with the tissue inhibitor of metalloproteinases 2, transforming growth factor-beta (TGF- $\beta$ ), alpha-smooth muscle actin ( $\alpha$-SMA), all of which were attenuated by thioctic acid supplementation $[15,24]$. The study showed that attenuation of the mitogen-activated protein kinase (MAPK) signaling pathway after treatment with thioctic acid may help limit the development of DCM. In addition, these results, together with a good safety profile and excellent tolerance of thioctic acid in humans, show that thioctic acid may have potential benefits in the management of DCM by mitigating mitochondrial oxidative stress, remodeling the ECM and activating MAPK [28].
Regarding the use of ALA, we can say that a previous study showed that high doses of ALA reduce body weight in obese people [29], the treatment being associated with the loss of body fat, which is mediated by suppressing the activity of adenosine monophosphate (AMP)-activated protein kinase (AMPK) in the hypothalamus [30]. In the animal model of STZ-induced DM, ALA did not attenuate weight loss in control animals [31]. Regarding the side effects of ALA, previous studies did not show any serious side effects [31]. Recent studies have also shown that LVEF, LV remodeling, inflammation and fibrosis have been alleviated by the administration of ALA [32].

Regarding vitamin B complex, only thiamine and pyridoxine were used in our study. Thiamine has an important role in carbohydrate metabolism [33-35]. Complications of thiamine deficiency in diabetes include increased oxidative stress, hyperglycemia-induced cell damage and endothelial dysfunction. Animal studies have shown that thiamine reduces activity through hexosamine, which may lead to decreased insulin resistance [33]. Thiamine supplementation also prevents the formation of AGEs, which can cause vascular damage by altering extra- and intracellular proteins. In vitro studies have shown that thiamine administration reduces protein kinase $\mathrm{C}$ activation in glomeruli $[34,35]$. Thiamine has also been shown to reduce the activation of the polyol pathway of glucose metabolism, thus reducing oxidative stress by decreasing nicotinamide adenine dinucleotide phosphate, reduced form (NADPH) [33-35]. Last but not least, vitamin B6 (pyridoxine) is a water-soluble vitamin that is also found in whole grains, potatoes, legumes, nuts, fish and others [36]. It is an essential vitamin and participates in many enzymatic reactions, including the formation of neurotransmitters, protein metabolism, and the conversion of homocysteine to methionine and tryptophan to niacin [37]. Pyridoxine is subsequently converted to pyridoxal 5 '-phosphate (PLP) and pyridoxamine, which function as coenzymes for the transaminase reaction [38]. Supplementation of vitamin B6 and its active metabolite (PLP) has been reported by studies to reduce complications associated with diabetes, coronary heart disease, hypertension, neurodegenerative disorders, and aging [39-41].

\section{a Conclusions}

Administration of ALA and vitamin B complex in mice with STZ-induced DM, decreases myocardial fibrosis and polysaccharide accumulation and prevents severe deterioration of systolic and diastolic heart function.

\section{Conflict of interests}

The authors declare that they have no conflict of interests.

\section{References}

[1] Saeedi P, Petersohn I, Salpea P, Malanda B, Karuranga S, Unwin N, Colagiuri S, Guariguata L, Motala AA, Ogurtsova K, Shaw JE, Bright D, Williams R; IDF Diabetes Atlas Committee. Global and regional diabetes prevalence estimates for 2019 and projections for 2030 and 2045: results from the International Diabetes Federation Diabetes Atlas, $9^{\text {th }}$ edition. Diabetes Res Clin Pract, 2019, 157:107843. https://doi.org/10.1016/j.diab res.2019.107843 PMID: 31518657 
[2] Petersmann A, Müller-Wieland D, Müller UA, Landgraf R, Nauck M, Freckmann G, Heinemann L, Schleicher E. Definition, classification and diagnosis of diabetes mellitus. Exp Clin Endocrinol Diabetes, 2019, 127(S 01):S1-S7. https://doi.org/ 10.1055/a-1018-9078 PMID: 31860923

[3] Murtaza G, Virk HUH, Khalid M, Lavie CJ, Ventura H, Mukherjee D, Ramu V, Bhogal S, Kumar G, Shanmugasundaram M, Paul TK. Diabetic cardiomyopathy - a comprehensive updated review. Prog Cardiovasc Dis, 2019, 62(4):315-326. https://doi.org/10.1016/j.pcad.2019.03.003 PMID: 30922976

[4] Ali TM, Abo-Salem OM, El Esawy BH, El Askary A. The potential protective effects of diosmin on streptozotocin-induced diabetic cardiomyopathy in rats. Am J Med Sci, 2020, 359(1): 32-41. https://doi.org/10.1016/j.amjms.2019.10.005 PMID: 31902439

[5] Aneja A, Tang WH, Bansilal S, Garcia MJ, Farkouh ME. Diabetic cardiomyopathy: insights into pathogenesis, diagnostic challenges, and therapeutic options. Am J Med, 2008, 121(9): 748-757. https://doi.org/10.1016/j.amjmed.2008.03.046 PMID: 18724960

[6] Rubler S, Dlugash J, Yuceoglu YZ, Kumral T, Branwood AW, Grishman A. New type of cardiomyopathy associated with diabetic glomerulosclerosis. Am J Cardiol, 1972, 30(6):595602. https://doi.org/10.1016/0002-9149(72)90595-4 PMID: 4263660 PMCID: PMC3969857

[7] Bugger H, Abel ED. Molecular mechanisms of diabetic cardiomyopathy. Diabetologia, 2014, 57(4):660-671. https://doi. org/10.1007/s00125-014-3171-6 PMID: 24477973 PMCID: PMC3969857

[8] Jia G, Whaley-Connell A, Sowers JR. Diabetic cardiomyopathy a hyperglycaemia- and insulin-resistance-induced heart disease. Diabetologia, 2018, 61(1):21-28. https://doi.org/10.1007/s00 125-017-4390-4 PMID: 28776083 PMCID: PMC5720913

[9] Jia G, Hill MA, Sowers JR. Diabetic cardiomyopathy: an update of mechanisms contributing to this clinical entity. Circ Res, 2018, 122(4):624-638. https://doi.org/10.1161/CIRCRESAHA. 117.311586 PMID: 29449364 PMCID: PMC5819359

[10] Dillmann WH. Diabetic cardiomyopathy. Circ Res, 2019, 124(8) 1160-1162. https://doi.org/10.1161/CIRCRESAHA.118.314665 PMID: 30973809 PMCID: PMC6578576

[11] Borghetti G, von Lewinski D, Eaton DM, Sourij H, Houser SR, Wallner M. Diabetic cardiomyopathy: current and future therapies. Beyond glycemic control. Front Physiol, 2018, 9:1514. https://doi.org/10.3389/fphys.2018.01514 PMID: 30425649 PMCID: PMC6218509

[12] Riehle C, Bauersachs J. Of mice and men: models and mechanisms of diabetic cardiomyopathy. Basic Res Cardiol, 2018, 114(1):2. https://doi.org/10.1007/s00395-018-0711-0 PMID: 30443826 PMCID: PMC6244639

[13] Paolillo S, Marsico F, Prastaro M, Renga F, Esposito L, De Martino F, Di Napoli P, Esposito I, Ambrosio A, lanniruberto M, Mennella R, Paolillo R, Gargiulo P. Diabetic cardiomyopathy: definition, diagnosis, and therapeutic implications. Heart Fail Clin, 2019, 15(3):341-347. https://doi.org/10.1016/j.hfc.2019. 02.003 PMID: 31079692

[14] Shay KP, Moreau RF, Smith EJ, Smith AR, Hagen TM. Alphalipoic acid as a dietary supplement: molecular mechanisms and therapeutic potential. Biochim Biophys Acta, 2009, 1790(10):1149-1160. https://doi.org/10.1016/j.bbagen.2009. 07.026 PMID: 19664690 PMCID: PMC2756298

[15] Lee JE, Yi CO, Jeon BT, Shin HJ, Kim SK, Jung TS, Choi JY, Roh GS. $\alpha$-Lipoic acid attenuates cardiac fibrosis in Otsuka Long-Evans Tokushima fatty rats. Cardiovasc Diabetol, 2012 11:111. https://doi.org/10.1186/1475-2840-11-111 PMID: 22992429 PMCID: PMC3558371

[16] Singh HP, Bowman RH. Effect of DL-alpha-lipoic acid on the citrate concentration and phosphofructokinase activity of perfused hearts from normal and diabetic rats. Biochem Biophys Res Commun, 1970, 41(3):555-561. https://doi.org/10.1016/ 0006-291x(70)90048-3 PMID: 4249179

[17] Ou P, Tritschler HJ, Wolff SP. Thioctic (lipoic) acid: a therapeutic metal-chelating antioxidant? Biochem Pharmacol, 1995, 50(1): 123-126. https://doi.org/10.1016/0006-2952(95)00116-h PMID: 7605337

[18] Vasdev S, Ford CA, Parai S, Longerich L, Gadag V. Dietary lipoic acid supplementation prevents fructose-induced hypertension in rats. Nutr Metab Cardiovasc Dis, 2000, 10(6):339 346. PMID: 11302009
[19] DiNicolantonio JJ, Liu J, O'Keefe JH. Thiamine and cardiovascular disease: a literature review. Prog Cardiovasc Dis 2018, 61(1):27-32. https://doi.org/10.1016/j.pcad.2018.01.009 PMID: 29360523

[20] Eshak ES, Arafa AE. Thiamine deficiency and cardiovascular disorders. Nutr Metab Cardiovasc Dis, 2018, 28(10):965-972. https://doi.org/10.1016/j.numecd.2018.06.013 PMID: 30143411

[21] Dhalla NS, Takeda S, Elimban V. Mechanisms of the beneficial effects of vitamin B6 and pyridoxal 5-phosphate on cardiac performance in ischemic heart disease. Clin Chem Lab Med, 2013, 51(3):535-543. https://doi.org/10.1515/cclm-2012-0553 PMID: 23314545

[22] Silva E, Natali AJ, Silva MF, Gomes GJ, Cunha DNQ, Ramos RMS, Toledo MM, Drummond FR, Belfort FG, Novaes RD, Maldonado IRSC. Ventricular remodeling in growing rats with experimental diabetes: the impact of swimming training. Pathol Res Pract, 2013, 209(10):618-626. https://doi.org/10. 1016/j.prp.2013.06.009 PMID: 23910625

[23] Spillmann F, Van Linthout S, Schultheiss HP, Tschöpe C. Cardioprotective mechanisms of the kallikrein-kinin system in diabetic cardiopathy. Curr Opin Nephrol Hypertens, 2006, 15(1):22-29. https://doi.org/10.1097/01.mnh.0000199009.567 99.2b PMID: 16340662

[24] Li CJ, Lv L, Li H, Yu DM. Cardiac fibrosis and dysfunction in experimental diabetic cardiomyopathy are ameliorated by alpha-lipoic acid. Cardiovasc Diabetol, 2012, 11:73. https:// doi.org/10.1186/1475-2840-11-73 PMID: 22713251 PMCID: PMC3472273

[25] Ryu SH, Park EY, Kwak S, Heo SH, Ryu JW, Park JH, Choi KC, Lee SW. Protective effect of $\alpha$-lipoic acid against radiationinduced fibrosis in mice. Oncotarget, 2016, 7(13):15554-15565. https://doi.org/10.18632/oncotarget.6952 PMID: 26799284 PMCID: PMC4941260

[26] Brunner F, Brás-Silva C, Cerdeira AS, Leite-Moreira AF. Cardiovascular endothelins: essential regulators of cardiovascular homeostasis. Pharmacol Ther, 2006, 111(2):508531. https://doi.org/10.1016/j.pharmthera.2005.11.001 PMID: 16457892

[27] Parim B, Sathibabu Uddandrao VV, Saravanan G. Diabetic cardiomyopathy: molecular mechanisms, detrimental effects of conventional treatment, and beneficial effects of natural therapy. Heart Fail Rev, 2019, 24(2):279-299. https://doi.org/ 10.1007/s10741-018-9749-1 PMID: 30349977

[28] Ghibu S, Richard C, Vergely C, Zeller M, Cottin Y, Rochette L. Antioxidant properties of an endogenous thiol: alpha-lipoic acid, useful in the prevention of cardiovascular diseases. J Cardiovasc Pharmacol, 2009, 54(5):391-398. https://doi.org/ 10.1097/fjc.0b013e3181be7554 PMID: 19998523

[29] Koh EH, Lee WJ, Lee SA, Kim EH, Cho EH, Jeong E, Kim DW, Kim MS, Park JY, Park KG, Lee HJ, Lee IK, Lim S, Jang HC, Lee $\mathrm{KH}$, Lee KU. Effects of alpha-lipoic acid on body weight in obese subjects. Am J Med, 2011, 124(1):85.e1-85.e8. https://doi.org/10.1016/j.amjmed.2010.08.005 PMID: 21187189

[30] Kim MS, Park JY, Namkoong C, Jang PG, Ryu JW, Song HS, Yun JY, Namgoong IS, Ha J, Park IS, Lee IK, Viollet B, Youn JH, Lee HK, Lee KU. Anti-obesity effects of alpha-lipoic acid mediated by suppression of hypothalamic AMP-activated protein kinase. Nat Med, 2004, 10(7):727-733. https://doi.org/ 10.1038/nm1061 PMID: 15195087

[31] Cremer DR, Rabeler R, Roberts A, Lynch B. Long-term safety of alpha-lipoic acid (ALA) consumption: a 2-year study. Regul Toxicol Pharmacol, 2006, 46(3):193-201. https://doi.org/10. 1016/j.yrtph.2006.06.003 PMID: 16899332

[32] Wang HT, Liu CF, Tsai TH, Chen YL, Chang HW, Tsai CY, Leu S, Zhen YY, Chai HT, Chung SY, Chua S, Yen CH, Yip HK. Effect of obesity reduction on preservation of heart function and attenuation of left ventricular remodeling, oxidative stress and inflammation in obese mice. J Transl Med, 2012, 10:145. https://doi.org/10.1186/1479-5876-10-145 PMID: 22784636 PMCID: PMC3551744

[33] Jermendy G. Evaluating thiamine deficiency in patients with diabetes. Diab Vasc Dis Res, 2006, 3(2):120-121. https:// doi.org/10.3132/dvdr.2006.014 PMID: 17058632

[34] Page GLJ, Laight D, Cummings MH. Thiamine deficiency in diabetes mellitus and the impact of thiamine replacement on glucose metabolism and vascular disease. Int J Clin Pract, 2011, 65(6):684-690. https://doi.org/10.1111/j.1742-1241.2011. 02680.x PMID: 21564442 
[35] Raval AD, Thakker D, Rangoonwala AN, Gor D, Walia R. Vitamin $B$ and its derivatives for diabetic kidney disease. Cochrane Database Syst Rev, 2015, 1:CD009403. https:// doi.org/10.1002/14651858.CD009403.pub2 PMID: 25579852

[36] Deshmukh SV, Prabhakar B, Kulkarni YA. Water soluble vitamins and their role in diabetes and its complications. Curr Diabetes Rev, 2020, 16(7):649-656. https://doi.org/10.2174/ 1573399815666190916114040 PMID: 31526351

[37] Fairfield KM, Fletcher RH. Vitamins for chronic disease prevention in adults: scientific review. JAMA, 2002, 287(23): 3116-3126. https://doi.org/10.1001/jama.287.23.3116 PMID: 12069675

[38] Selhub J, Jacques PF, Wilson PW, Rush D, Rosenberg IH. Vitamin status and intake as primary determinants of homocysteinemia in an elderly population. JAMA, 1993, 270(22):
2693-2698. https://doi.org/10.1001/jama.1993.03510220049 033 PMID: 8133587

[39] Rogers KS, Mohan C. Vitamin B6 metabolism and diabetes. Biochem Med Metab Biol, 1994, 52(1):10-17. https://doi.org/ 10.1006/bmmb.1994.1027 PMID: 7917462

[40] Tehlivets O. Homocysteine as a risk factor for atherosclerosis: is its conversion to $S$-adenosyl- $L$-homocysteine the key to deregulated lipid metabolism? J Lipids, 2011, 2011:702853. https://doi.org/10.1155/2011/702853 PMID: 21837278 PMCID: PMC3151505

[41] Berceanu M, Mirea O, Donoiu I, Militaru C, Săftoiu A, Istrătoaie O. Myocardial function assessed by multi-layered two-dimensional speckle tracking analysis in asymptomatic young subjects with diabetes mellitus type 1. Cardiology, 2020, 145(2):80-87. https://doi.org/10.1159/000504532 PMID: 31825945

\section{Corresponding author}

lonuţ Donoiu, Associate Professor, MD, PhD, Department of Cardiology, University of Medicine and Pharmacy of Craiova, 2 Petru Rareş Street, 200349 Craiova, Romania; Phone +40746-126 669, Fax +40251-426 688, e-mail: ionut.donoiu@umfcr.ro

Received: March 22, 2020

Accepted: November 27, 2020 